\title{
Sample Correction for Digitally Controlled Boost PFC Converters Operating in both CCM and DCM
}

\author{
Koen De Gussemé*, David M. Van de Sype, Alex P. Van den Bossche and Jan A. Melkebeek \\ Electrical Energy Laboratory \\ Department of Electrical Energy, Systems and Automation, Ghent University \\ Sint-Pietersnieuwstraat 41, B-9000 Gent, Belgium \\ *E-mail: koen.degusseme@rug.ac.be
}

\begin{abstract}
PFC converters for the higher power range are commonly designed for continuous conduction mode. Nevertheless, at light load, DCM will appear close to the crossover of the line voltage, causing the converter to switch between CCM and DCM. As a result of this switching during a line period, the converter dynamics change abrubtly, yielding input current distortion. Moreover, if digital control is applied, another source of input current distortion is posed by the sampling algorithm. After all, the sampling algorithm is only designed to produce samples of the averaged input current in CCM. In this paper, after a study of the input current distortion caused by the sampling algorithm, a correction factor is derived to compensate for the error on the input current samples. The theoretical results are verified experimentally by using a digitally controlled boost PFC converter.
\end{abstract}

\section{INTRODUCTION}

In order to supply DC-applications from the AC-grid, a rectifier is required. If low harmonic content of the input current is required, power factor correction (PFC) converters, consisting of a bridge rectifier and a switching DC-DC converter, are employed as rectifiers. For low power applications, DC-DC converters such as buck-boost, boost, SEPIC or Ćuk converters are often operated in discontinuous conduction mode (DCM). After all, these converters require only a single control loop [1]-[3] to assure both a resistive input and a constant output voltage. Nevertheless, due to high device stresses and problems with conducted emission, the use of converters operating in DCM is limited to the low power range $(<250 \mathrm{~W})$. For the higher power range, converters are commonly designed for continuous conduction mode (CCM). Nevertheless, at light load, DCM will appear close to the crossover of the line voltage [4], causing the converter to switch between CCM and DCM (this mode of operation will be referred to as mixed conduction mode or MCM). As a result of this switching during a line period, the converter dynamics change abrubtly [3], [5], yielding input current distortion. If digital control is applied, sampling of the input current is usually necessary [6]-[10], although some attempts are made to control a boost PFC converter without instantaneous measurement of the input current [11]. In the case of input current sampling, another source of input current distortion in MCM is posed by the sampling algorithm. After all, the sampling algorithm is only designed to produce samples of the averaged input current in CCM [7], [9], [10]. In this paper, the conditions for MCM op- eration are determined and the input current distortion caused by the sampling algorithm, is analysed, yielding a correction factor to compensate for the error on the input current samples. Eventually, all theoretical results are verified experimentally by using a digitally controlled boost PFC converter.

\section{Digital Control of PFC Converters Operated IN CCM}

Fig. 1 shows the circuit diagram of a digitally controlled boost PFC converter. For the purpose of digital control, the analog control variables (the input current $i_{L}(t)$, the input voltage $v_{i n}(t)$ and the output voltage $\left.v_{o}(t)\right)$ are converted into dimensionless digital quantities $\left(i_{L, n}, v_{i n, n}\right.$ and $\left.v_{o, n}\right)$ after dividing by their respective reference values $I_{L}^{\text {ref }}, V_{i n}^{\text {ref }}$ and $V_{o}^{r e f}$. A first PI-controller acts on the duty-ratio $d_{n}$ of the switch $S$, comparing the input current sample $i_{L, n}$ with its desired value $i_{L, n}^{*}$. The latter, the product of the sampled input voltage $v_{i n, n}$ and the desired input conductance $g_{e, n}$ of the converter, is provided by a second PI-controller controlling the output voltage. As these controllers use samples to perform their control actions, it is important that an accurate representation of the sampled waveforms is obtained. As the input current contains a switching ripple with large magnitude (Fig. 2), due to the switching of the boost converter, an accurate reconstruction of the signal is only possible when the sampling frequency is sufficiently higher than the switching frequency. This is hard to accomplish since the switching frequency is already high $\left(f_{s w}>20 \mathrm{kHz}\right)$ leaving the processor not enough cycles to perform the necessary calculations for control. On the other hand, using a lower sampling frequency will cause aliasing. Therefore, the sampling frequency is often chosen equal to the switching frequency. As the input current waveform is now represented by one sample each switching cycle, it is very important to choose the right sampling instant [10]. This instant is commonly chosen in the middle of the rising edge of the input current waveform. This algorithm is called rising edge sampling (RES) [7], [9], [10].

\section{Conditions for Mixed CurRent Mode OPERATION}

\section{A. Boost converters operated as DC-DC converters}

When operated as DC-DC converters, boost converters operate in border mode when the averaged input current is 


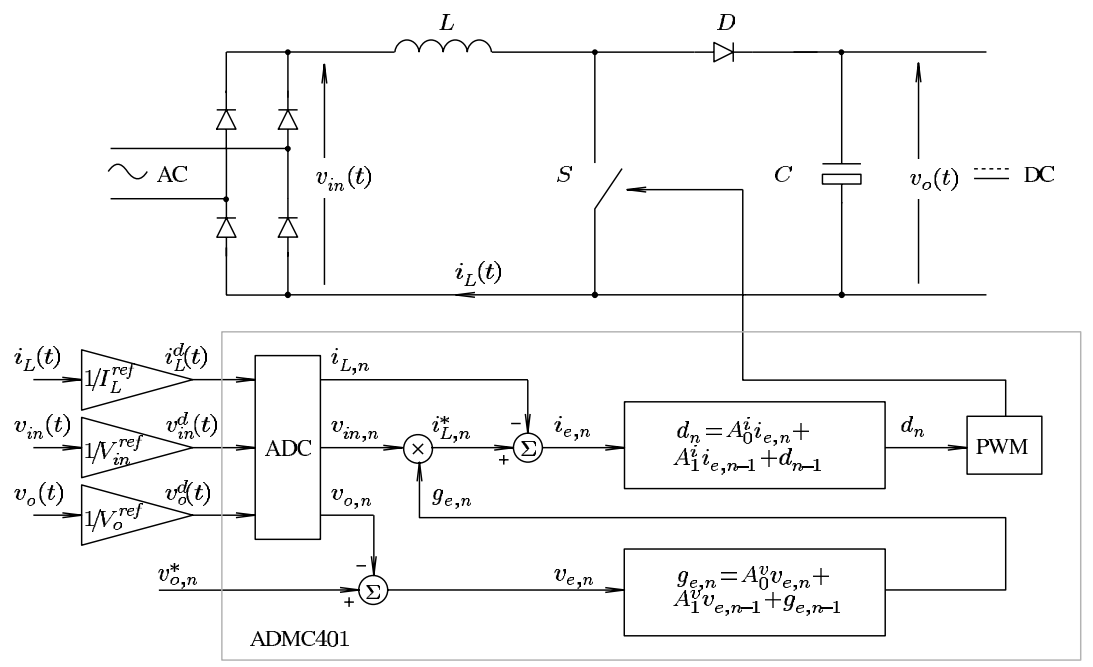

Fig. 1. A digitally controlled boost PFC converter

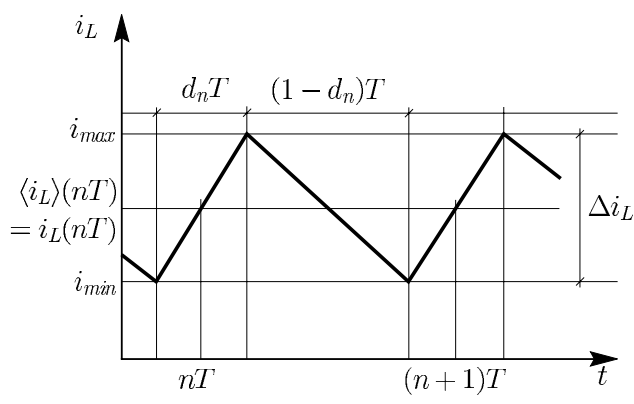

Fig. 2. Input current waveform $i_{L}(t)$ for $\mathbf{C C M}$

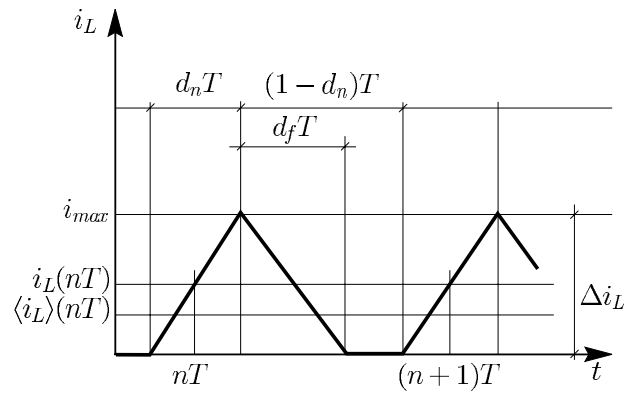

Fig. 3. Input current waveform $i_{L}(t)$ for DCM equal to:

$$
\left\langle i_{L}\right\rangle=\frac{d_{n} T v_{i n}}{2 L} .
$$

Higher values of the averaged input current will result in CCM operation (Fig. 2), while lower values will lead to DCM operation (Fig. 3).

\section{B. Assumptions}

For the purpose of power factor correction, the duty-ratio $d_{n}$, the input voltage $v_{i n}$ and the averaged input current $\left\langle i_{L}\right\rangle$ are time dependent, causing the boundary between DCM and CCM to vary during a grid cycle. In order to obtain a mathematical expression for these boundaries, some assumptions are made:

- The input voltage is a rectified sine wave

$$
v_{\text {in }}(t)=\widehat{V}_{g}|\sin (\omega t)| \text {. }
$$

- The input current controller forces the obtained samples to track the input voltage waveform perfectly,

$$
i_{L}(n T)=g_{e}(n T) v_{i n}(n T)
$$

where $g_{e}$ represents the desired input conductance of the converter.

- The output capacitor $C_{o}$ of the converter has a large capacitance value, leading to a constant output voltage $v_{o}(t)=V_{o}$. As a result, the desired input conductance $g_{e}(t)$ of the converter will not be altered by the output voltage controller, so it can be assumed to have a constant value $G_{e}$. Expression (3) can now be written as

$$
i_{L}(n T)=G_{e} v_{i n}(n T) .
$$

\section{Boundaries of the Mixed Conduction Mode}

With these assumptions, (1) can be transferred into a condition for CCM operation during the entire line cycle. Therefore, the condition

$$
\left\langle i_{L}\right\rangle(n T)=G_{e} v_{i n}(n T)>\frac{d_{n} T v_{i n}}{2 L}
$$

must be met during the entire grid period. As a result, a condition for the input conductance $G_{e}$ can be derived as a function of the time dependent duty-ratio $d_{n}$ :

$$
G_{e}>\frac{d_{n} T}{2 L}
$$

In $\mathrm{CCM}$, the duty-ratio is given by

$$
d_{n}=1-\frac{v_{i n}(n T)}{v_{o}(n T)}=1-\frac{\widehat{V}_{g}}{V_{o}} \sin (\omega n T) .
$$

The maximum value of the duty-ratio $d_{n}$ is reached at the zero-crossing of the input voltage, where it becomes unity, resulting in

$$
G_{e}>\frac{T}{2 L} .
$$

As for CCM operation the averaged input current has the same waveform as the input voltage, a rectified sine wave, an equivalent condition can be derived for the desired input power,

$$
P_{i n}=G_{e} V_{g}^{2}>\frac{T}{2 L} V_{g}^{2},
$$

with $V_{g}$ the rms value of the input voltage, $V_{g}=\frac{\widehat{V}_{g}}{\sqrt{2}}$. 


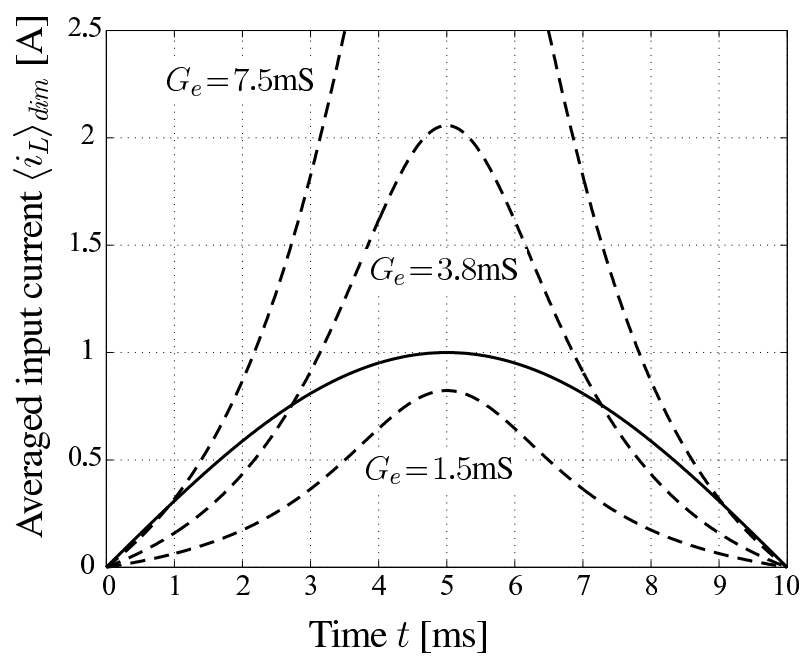

Fig. 4. Dimensionless averaged input current waveforms for CCM (solid line) and DCM (dashed lines)

The condition for the boost converter to operate in DCM can be obtained analogously. The condition becomes

$$
G_{e}<\frac{d_{n} T}{2 L} .
$$

By taking (7) into account, the boundary can be calculated as

$$
G_{e}<\frac{T}{2 L}\left(1-\frac{\widehat{V}_{g}}{V_{o}}\right)
$$

Since the averaged input current waveform in DCM is no longer equal to the input voltage waveform, as will be described below, the effective value of the input conductance $G_{e}$ will be smaller than its programmed value. As a result, no condition for the input power can be derived from (11).

\section{VAlue of the InPUT CuRREnt SAmple}

To obtain the value of the input current samples, we assume that the converter operates near equilibrium with a slowly moving quiescent operating point. This implies that the input voltage $v_{i n}(t)$ and output voltage $v_{o}(t)$, as well as the input current ripple $\Delta i_{L}(t)$ and the averaged input current $\left\langle i_{L}\right\rangle(t)$ (averaged over one switching cycle), exhibit only small variations during one switching period, even though the input current $i_{L}(t)$ alters quickly in a switching period (Fig. 2). Fig. 2 shows clearly that, under these conditions, the sample $i_{L}(n T)$, taken in the middle of the rising edge of the input current waveform, is equal to the averaged input current $\left\langle i_{L}\right\rangle(n T)$, when the converter is operating in CCM:

$$
\left\langle i_{L}\right\rangle(n T)=i_{L}(n T)
$$

For DCM, the input current sample, taken in the middle of the rising edge, is given by (Fig. 3)

$$
i_{L}(n T)=\frac{i_{\max }(n T)}{2} .
$$

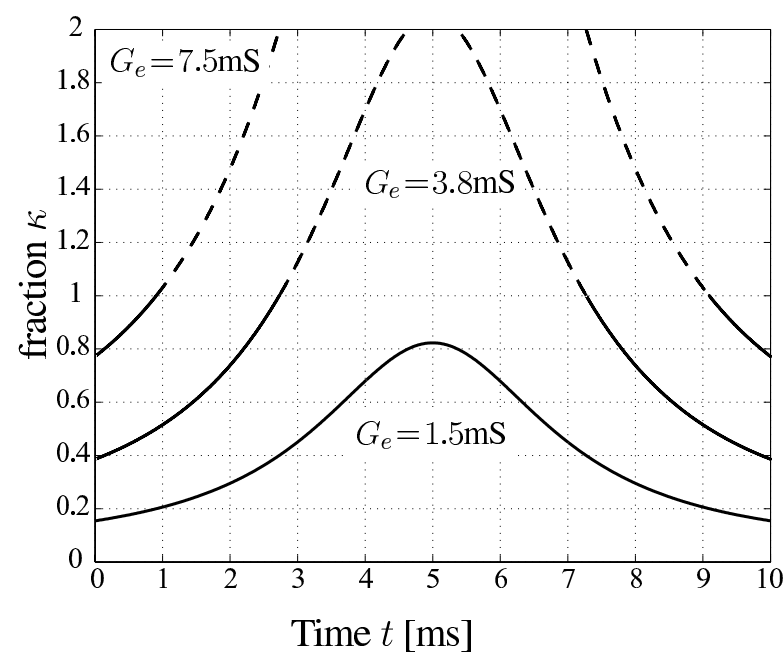

Fig. 5. Theoretical waveform of the fraction $\kappa$ corresponding with DCM operation

As the input current only differs from zero in a period $\left(d_{n}+d_{f, n}\right) T$, the averaged input current can now be calculated as (Fig. 3)

$$
\left\langle i_{L}\right\rangle(n T)=\frac{i_{\max }(n T)}{2} \frac{\left(d_{n}+d_{f, n}\right) T}{T} .
$$

Combining (13) and (14) leads to

$$
\left\langle i_{L}\right\rangle(n T)=i_{L}(n T)\left(d_{n}+d_{f, n}\right) \triangleq \kappa(n T) i_{L}(n T),
$$

where $\kappa(n T)$ represents the fraction of the switching period where the current is not zero, and consists of the rising edge fraction $d_{n} T$ and the falling edge fraction $d_{f, n} T$. As a result, for CCM, $\kappa(n T)$ equals unity, while for DCM it is smaller than one. This means that the input current sample is smaller than the averaged input current, which will cause input current distortion.

In order to obtain a mathematical expression for the fraction $\kappa(n T), d_{f, n}$ is expressed as a function of $v_{i n}, v_{o}$ and $d_{n}$. As the slope of the inductor current $i_{L}$ is proportional to the voltage across the inductor, the rising edge fraction $d_{n} T$ and the falling edge fraction $d_{f, n} T$ are related by the following expression:

$$
d_{n} T v_{i n}=d_{f, n} T\left(v_{o}-v_{i n}\right) .
$$

Together with (15), this leads to

$$
\kappa(n T)=d_{n}+d_{f, n}=\frac{d_{n} v_{o}(n T)}{v_{o}(n T)-v_{i n}(n T)} .
$$

\section{InPUT CURRENT Distortion INDUCED By THE SAMPLING Algorithm IN MCM}

\section{A. The Averaged Input Current Waveform for CCM}

By assuming (4), the averaged input current can be calculated immediately, as for CCM the input current samples are equal to the averaged input current (12),

$$
\left\langle i_{L}\right\rangle(n T)=G_{e} v_{i n}(n T),
$$




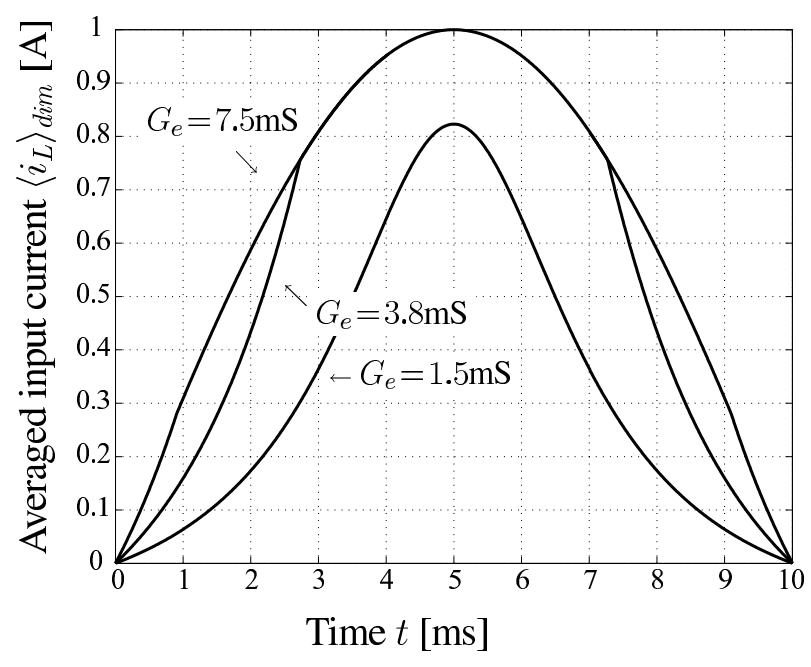

Fig. 6. Averaged input current waveform $\left\langle i_{L}\right\rangle(t)$

or with (2),

$$
\left\langle i_{L}\right\rangle(n T)=G_{e} \widehat{V}_{g}|\sin (\omega n T)| .
$$

The resulting waveform is shown in Fig. 4 (solid line) as a dimensionless quantity, given by

$$
\left\langle i_{L}\right\rangle_{\operatorname{dim}}(n T)=\frac{\left\langle i_{L}\right\rangle(n T)}{G_{e} \widehat{V}_{g}}=|\sin (\omega n T)| .
$$

\section{B. The Averaged Input Current Waveform for DCM}

As in DCM the input current sample $i_{L}(n T)$ is not equal to the averaged input current $\left\langle i_{L}\right\rangle(n T)$ (15), the averaged input current waveform will differ from (19). Expressing $i_{\max }$ as

$$
i_{\text {max }}(n T)=\frac{d_{n} T v_{\text {in }}(n T)}{L},
$$

the input current sample (13) can be written as

$$
i_{L}(n T)=\frac{d_{n} T}{2 L} v_{i n}(n T) .
$$

When both (4) and (22) have to be met, the duty-ratio should have a constant value

$$
d_{n}=\frac{2 L G_{e}}{T} .
$$

By substituting (23) in (17), the ratio $\kappa(n T)$ for DCM can be determined,

$$
\kappa(n T)=\frac{2 G_{e} L}{T} \frac{v_{o}(n T)}{v_{o}(n T)-v_{i n}(n T)} .
$$

Substituting (24) in (15), together with (4), directly yields an expression for the averaged input current in DCM,

$$
\left\langle i_{L}\right\rangle(n T)=\frac{2 G_{e} L}{T} \frac{v_{o}(n T)}{v_{o}(n T)-v_{i n}(n T)} G_{e} v_{i n}(n T),
$$

or, under the assumptions of section III-B,

$$
\left\langle i_{L}\right\rangle(n T)=\frac{2 G_{e} L}{T} \frac{V_{o}}{V_{o}-\widehat{V}_{g}|\sin (\omega n T)|} G_{e} \widehat{V}_{g}|\sin (\omega n T)| .
$$

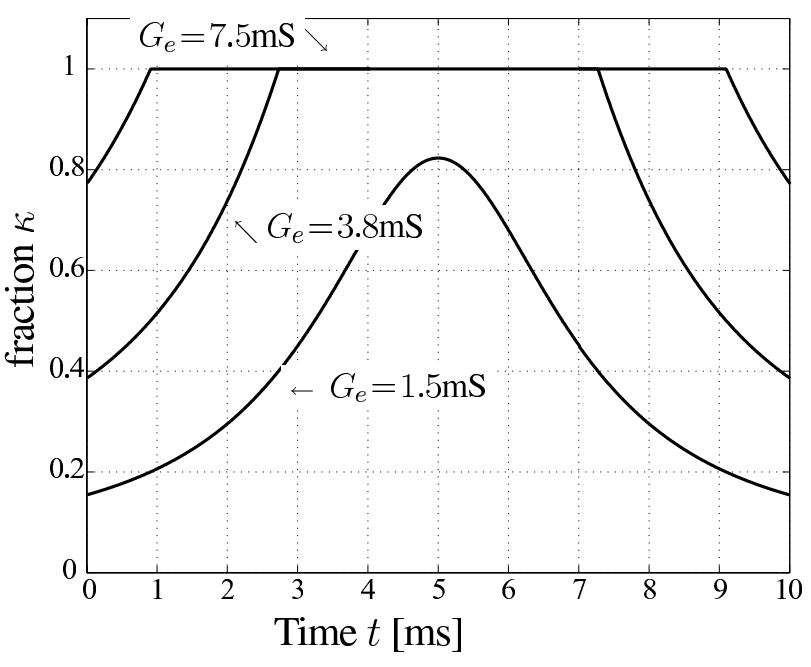

Fig. 7. Correction factor $\kappa(t)$

The dimensionless averaged input current $\left\langle i_{L}\right\rangle_{\operatorname{dim}}(n T)$ is given by

$$
\left\langle i_{L}\right\rangle_{\operatorname{dim}}(n T)=\frac{2 G_{e} L}{T} \frac{V_{o}}{V_{o}-\widehat{V}_{g}|\sin (\omega n T)|}|\sin (\omega n T)|,
$$

and is represented by the dashed lines in Fig. 4 for several values of $G_{e}$. The corresponding waveforms of $\kappa$, resulting from (24) are shown in Fig. 5. Values of $\kappa$ larger than one have no physical meaning, by definition (15) of $\kappa$ and will never appear. Therefore, these values are represented by a dashed line in Fig. 5.

\section{The Input Current Waveform for MCM}

As operation in MCM implies operation in CCM as well as in DCM, the total averaged input current waveform in MCM will be a combination of (19) and (26), shown in Figs. 4 and 5.

The fraction $\kappa$ reaches unity at the intersection of curves (19) and (26). As values of $\kappa$ smaller than unity correspond with DCM operation, DCM will only occur when (26) is smaller than (19), while CCM operation will occur in the remainder of the line period. In this CCM period $\kappa$ is unity, by definition (15). The resulting averaged input current waveform is shown in Fig. 6 for several values of the input conductance $G_{e}$. Fig. 7 shows the corresponding values of the fraction $\kappa$.

For high power levels, corresponding with larger values of the input conductance $G_{e}$, the averaged input current has a large amplitude, so (1) is met during the entire line cycle. As a result, the converter operates in CCM during the entire grid period, resulting in a perfect input current waveform. This is no longer true for low input power, where the converter operates in DCM during a part of the line period, inducing an important input current distortion. The waveforms of Figs. 4 to 7 were obtained using the converter parameters of the experimental setup described in section VII. 


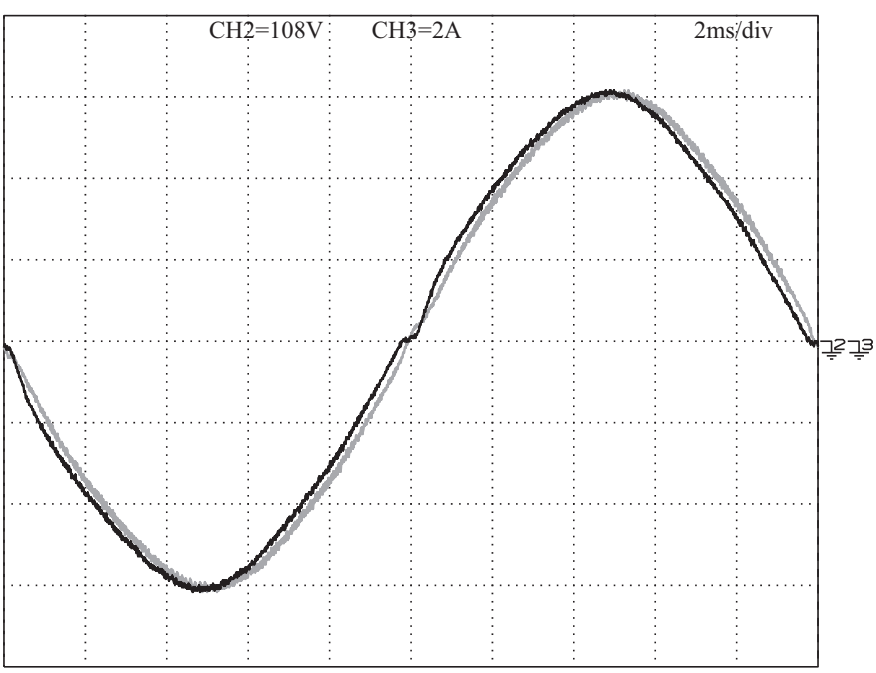

Fig. 8. Converter operating at $1 \mathrm{~kW}$ output power, black: averaged input current, gray: input voltage

\section{SAMPLE CORRECTION}

Since the relation between the input current samples $i_{L}(n T)$ and the averaged input current $\left\langle i_{L}\right\rangle(n T)$ is now determined for both DCM and CCM, the processor can be programmed to correct the input current samples for DCM by multiplying the dimensionless input current samples $i_{L, n}$ by a correction factor, the ratio $\kappa(17)$. As $v_{i n}$ and $v_{o}$ are sampled control variables and $d_{n}$ is the output of the digital controller, the processor has all the required data for calculating $\kappa$. The multiplication of the input current sample and the correction factor is valid in both CCM, where the correction factor is unity, and DCM. Hence, detection of DCM operation is not required, simplifying the digital control.

Using sample correction will lead to an averaged input current waveform which perfectly matches the input voltage waveform, when a perfect controller is assumed. As a result, operation in MCM will not imply increasing input current distortion anymore, allowing the converter to be operated at lower power.

\section{EXPERIMENTAL RESULTS}

For the experimental verification of the sample correction, a digitally controlled boost PFC converter with the circuit diagram of Fig. 1 and following characteristics has been employed:

$$
\begin{cases}V_{g}=230 \mathrm{~V}, \quad f_{g}=50 \mathrm{~Hz}, & T=19.6 \mu \mathrm{s} \\ V_{o}=400 \mathrm{~V}, & C=470 \mu \mathrm{F}, \quad L=1 \mathrm{mH}\end{cases}
$$

The converter is designed for operation at $1 \mathrm{~kW}$, yielding the input current waveform of Fig. 8. For a converter with these characteristics, the minimal value of the input power for CCM operation can be calculated using (9), resulting in $518 \mathrm{~W}$. Hence, the converter will operate in CCM during the entire grid cycle, when operated at $1 \mathrm{~kW}$, while DCM operation will appear near the crossover of the line voltage for a converter operating at $400 \mathrm{~W}$ output power (Fig. 9). For $400 \mathrm{~W}$ output

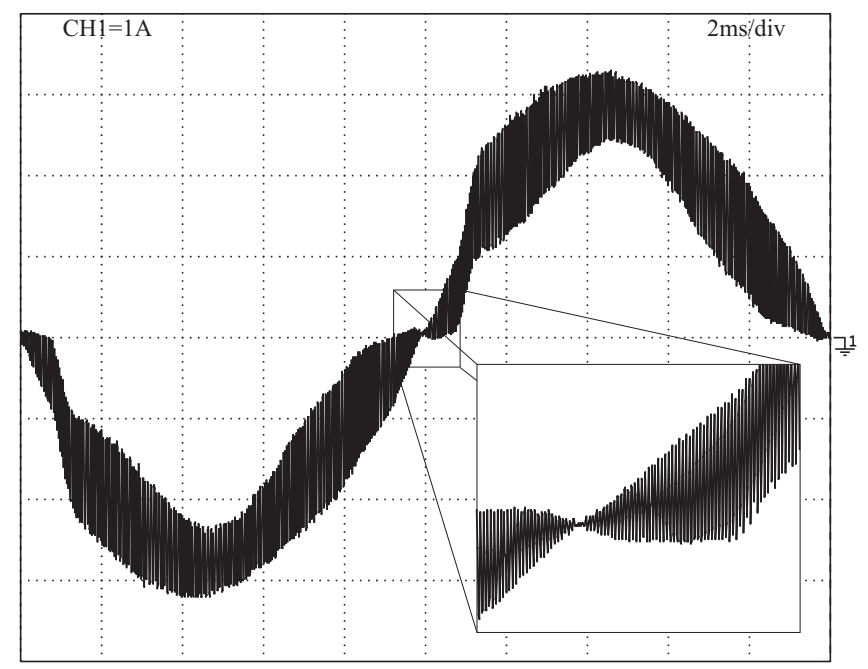

Fig. 9. Input current waveform for the converter operating at $400 \mathrm{~W}$ output power

power, the input current near the crossover is depicted in the inset of Fig. 9, revealing DCM operation during a small interval. The minimal value of $\kappa$ reached in this interval can be calculated using (24),

$$
\kappa_{\max }=\frac{2 G_{e} L}{T}=0.816,
$$

where the value of the input conductance, needed for operation at $400 \mathrm{~W}$ output power is equal to $8.32 \mathrm{mS}$. This value of the fraction $\kappa$ is still too close to unity to observe its influence on the converter waveform.

With decreasing values of the converter power level, the influence of the errors on the input current samples becomes more important. For an output power of $150 \mathrm{~W}$, the distortion of the averaged input current in discontinuous conduction mode is clearly visible in Fig. 10. The lower trace shows an analog representation of the input current error, which is used as input of the digital current controller. In DCM, this error is very small, which means that the value of the input current samples are close to the desired value of the input current. Nevertheless, the resulting averaged input current waveform differs from the input voltage waveform, as the obtained samples are too small.

When sample correction is applied, the averaged input current waveform in DCM has a better resemblance with the input voltage waveform, as shown in Fig. 11 for $150 \mathrm{~W}$ operation, although the total averaged input current waveform still contains an important amount of distortion. This is due to the sudden changes in converter dynamics, occuring when the converter switches between DCM and CCM. The lower trace of Fig. 11 shows the resulting input current error, fed to the input current controller. This error is now a correct representation of the effective input current error, but it is not zero as the controller is designed for the converter dynamics in $\mathrm{CCM}$.

If the power level is further decreased, the converter will operate in DCM during the entire grid cycle when the input 


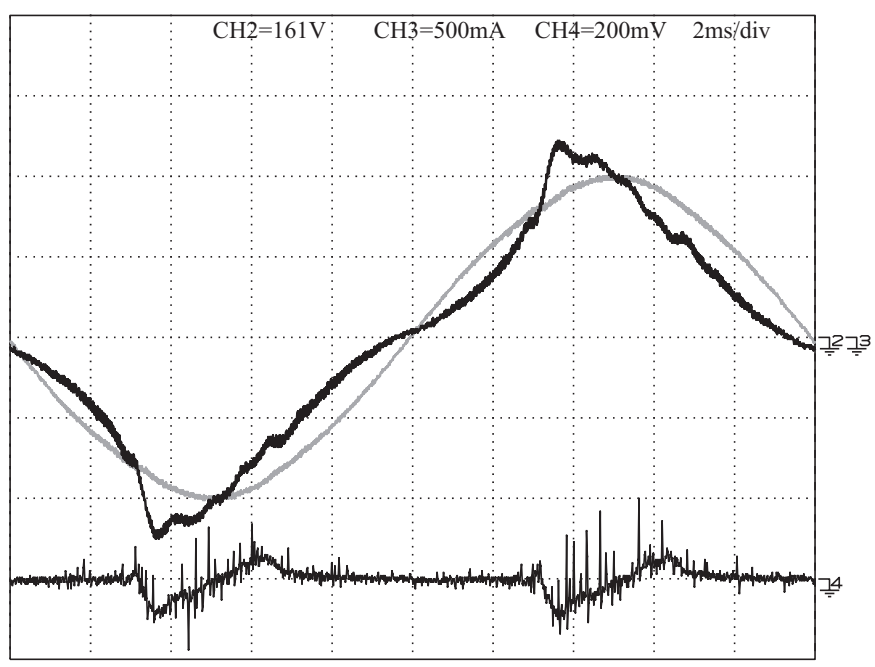

Fig. 10. Converter operating at $150 \mathrm{~W}$ without sample correction, upper traces, black: averaged input current, gray: input voltage, lower trace: input current error

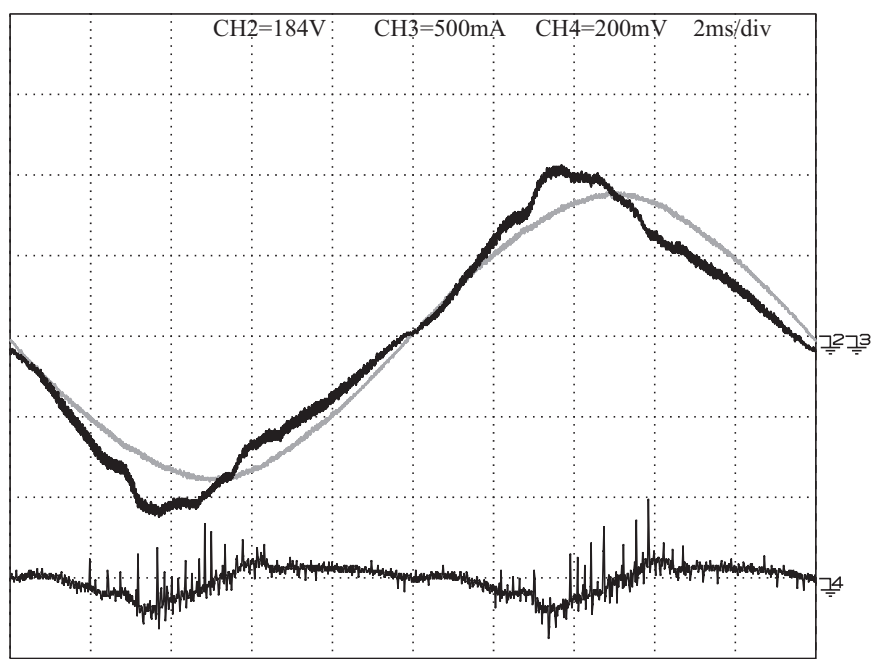

Fig. 11. Converter operating at $150 \mathrm{~W}$ with sample correction, upper traces, black: averaged input current, gray: input voltage, lower trace: input current error

power is lower than

$$
P_{\text {in }}<\frac{T}{2 L} V_{g}^{2}\left(1-\frac{\widehat{V}_{g}}{V_{o}}\right)=97.2 W
$$

when a sinusoidal averaged input current waveform is obtained. Fig. 12 shows that for $75 \mathrm{~W}$ output power the converter is still operating in MCM when no sample correction is applied. Since the sampled values of the input current overestimate the value of the averaged input current, (15), the effective input conductance is lower than its desired value. As a result, the converter input power will be less than its programmed value $G_{e} V_{g}^{2}$, leading to a low output capacitor voltage, so $G_{e}$ will be increased by the output voltage controller. This will cause the input current to cross the boundary between DCM and CCM near the top of the input voltage waveform. The

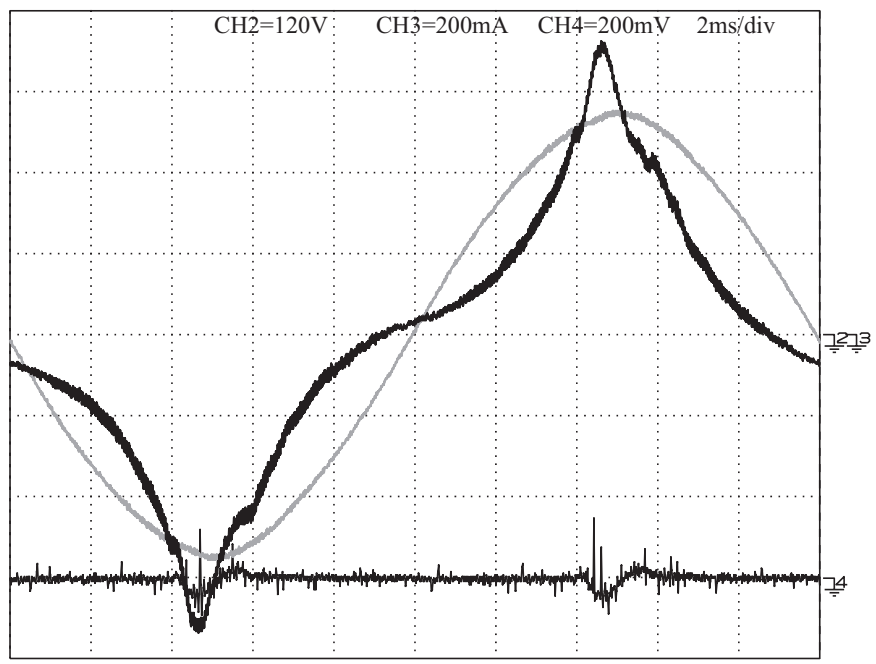

Fig. 12. Converter operating at $75 \mathrm{~W}$ without sample correction, upper traces, black: averaged input current, gray: input voltage, lower trace: input current error

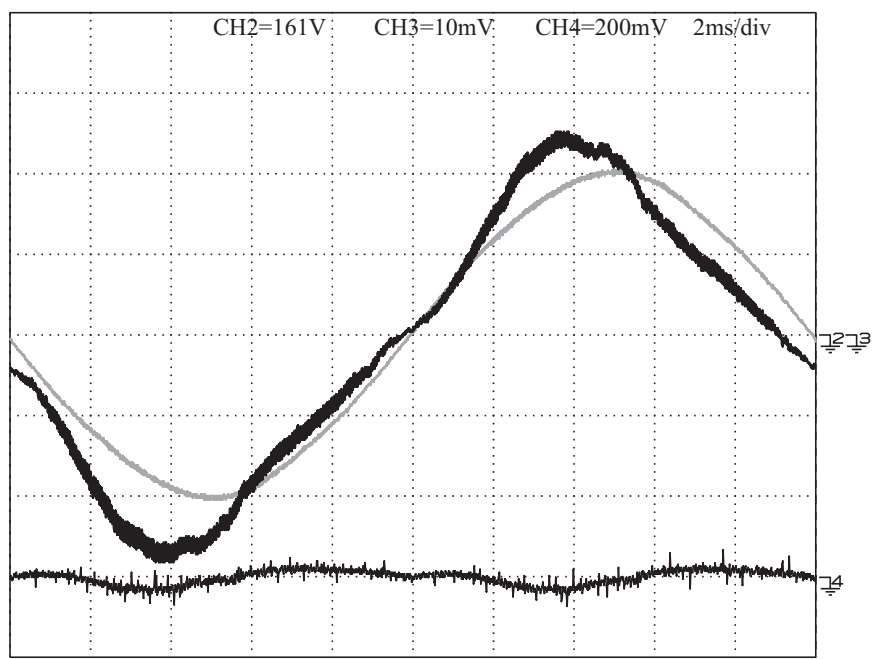

Fig. 13. Converter operating at $75 \mathrm{~W}$ with sample correction, upper traces, black: averaged input current, gray: input voltage, lower trace: input current error

averaged input current waveform does not match its desired value, although the input current error is very small in DCM.

Fig. 13 shows the input waveforms of the converter operating in DCM during the entire line period when operated at $75 \mathrm{~W}$ output power. The averaged input current waveform is still not perfectly matching the input voltage waveform, as the input current controller is designed for CCM operation. In Fig. 14, these converter waveforms are shown for an input current controller designed for DCM, resulting in a nearly sinusoidal averaged input current waveform.

\section{CONCLUSION}

Digitally controlled boost PFC converters, designed for operating in CCM, show input current distortion when operated at reduced power, as DCM appears near the crossover of the line voltage. This input current distortion is caused by the 


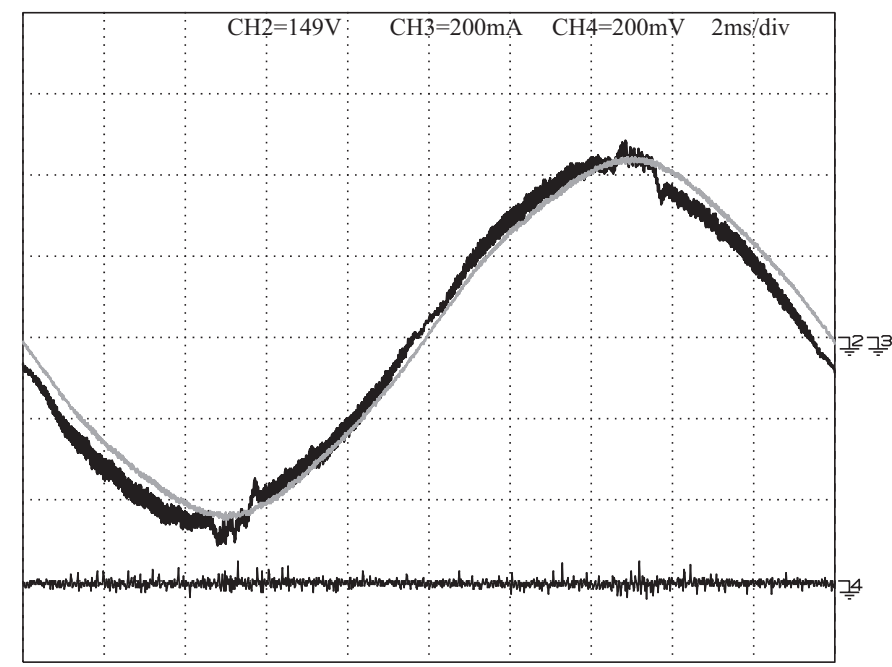

Fig. 14. Converter operating at $75 \mathrm{~W}$ with sample correction and $\mathrm{dcm}$ controller, upper traces, black: averaged input current, gray: input voltage, lower trace: input current error

change in converter dynamics in DCM and to erroneous input current sampling, since the sampling algorithm is designed to create input current samples in CCM. In this paper, the input current distortion, due to erroneous input current sampling when the rising edge sampling algorithm is used, was analysed, yielding a mathematical expression for the input current waveform. This expression was employed to derive a correction factor $\kappa$, used to compensate for the error on the input current samples. Since this correction factor $\kappa$ can be expressed as a function of the control variables, the processor can correct the samples of the input current to obtain a good estimate of the averaged input current.
The resulting improvement of the averaged input current waveform was verified experimentally on a test setup.

\section{REFERENCES}

[1] J. Sebastian, J.A. Martínez, J.M. Alonso, and J.A. Cobos, "Voltagefollower control in zero-current-switched quasi-resonant power factor preregulators," IEEE Trans. Power Electr., Vol. 13, No. 4, July 1998, pp. 727-738.

[2] D.S.L. Simonetti, J. Sebastian, and J. Uceda, "The discontinuous conduction mode sepic and ćuk power factor preregulators: analysis and design," IEEE Trans. Ind. Electr., Vol. 44, No. 5, Oct. 1997, pp. 630637.

[3] D.S.L. Simonetti, J.L.F. Vieira, and G.C.D. Sousa, "Modeling of the high-power-factor discontinuous boost rectifiers," IEEE Trans. Ind. Electr., Vol. 46, No. 4, Aug. 1999, pp. 788-795.

[4] J. Sebastian, J.A. Cobos, J.M. Lopera, and J. Uceda, "The determination of the boundaries between continuous and discontinuous conduction modes in pwm dc-to-dc converters used as power factor preregulators," IEEE Trans. Power Electr., Vol. 10, No. 5, Sept. 1995, pp. 574-582.

[5] V. Vorpérian, "Simplified analysis of pwm converters using model of pwm switch, part II: discontinuous conduction mode" IEEE Trans. Aero. Electr. Sys., Vol. 26, No. 3, May 1990, pp. 497-505.

[6] J. Zhou, Z. Lu, Z. Lin, Y. Ren, Z. Qian, and Y. Wang, "Novel sampling algorithm for DSP controlled 2kW PFC converter," IEEE Trans. Power Electr., Vol. 16, No. 2, March 2001, pp. 217-222. pp.185-192.

[7] S. Buso, P. Mattavelli, L. Rossetto, and G. Spiazzi, "Simple digital control improving dynamic performance of power factor preregulators," IEEE Trans. Power Electr., Vol. 13, No. 5, Sept. 1998, pp. 814-823.

[8] A.H. Mitwalli, S.B. Leeb, G.C. Verghese, and V.J. Thottuvelil, "An adaptive digital controller for a unity power factor converter," IEEE Trans. Power Electr., Vol. 11, No. 2, March 1996, pp. 374-382.

[9] K. De Gussemé, D.M. Van de Sype, and J.A.A. Melkebeek, "Design issues for digital control of boost power factor correction converters," Proc. of the IEEE Int. Symp. Ind. Electr., ISIE, 2002, pp. 731-736.

[10] D.M. Van de Sype, K. De Gussemé, and J.A.A. Melkebeek, "A sampling algortihm for digitally controlled boost PFC converters," Proc of the IEEE Power Electr. Spec. Conf., PESC, 2002, pp.1693-1698.

[11] S. Sivakumar, K. Natarajan, and R. Gudelewicz, "Control of power factor controlling boost converter without instantaneous measurement of input current," IEEE Trans. Power Electr., Vol. 10, No. 4, July 1995, pp. 435445 